\title{
Melanoma Cells Use Thy-1 (CD90) on Endothelial Cells for Metastasis Formation
}

\author{
Kathleen Schubert, ${ }^{*}$ Danny Gutknecht, ${ }^{*}$ Margarethe Köberle, ${ }^{\dagger}$ Ulf Anderegg, ${ }^{*}$ and Anja Saalbach ${ }^{*}$
}

From the Department of Dermatology, Venerology and Allergology, ${ }^{*}$ and the Institute of Clinical Immunology and Transfusion, ${ }^{\dagger}$ Medical Faculty of the Leipzig University, Leipzig, Germany

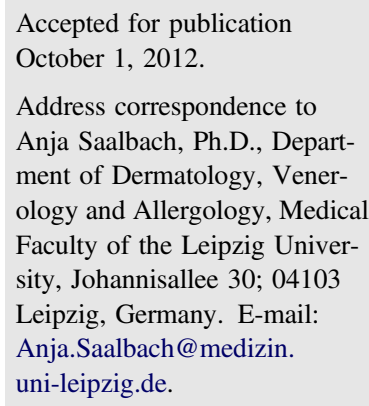

\begin{abstract}
The cell adhesion molecule Thy-1 (CD90) mediates the adhesion of melanoma cells to activated human endothelial cells (EC) via the interaction with the $\alpha v \beta 3$-integrin on the tumor cells in vitro. Here, we report a strong expression of Thy-1 on both blood vessel and lymphatic EC in melanoma and melanoma metastases. Vascular endothelial growth factor and tumor necrosis factor- $\alpha$ were identified as inducers of Thy- 1 expression on EC in vitro. The physiological role of Thy-1 for lymphogenic and hematogenic metastasis of melanoma cells was substantiated in an experimental metastasis model using B16/F10 melanoma cells. Mice lacking Thy-1 showed markedly diminished experimental lung metastasis after injection of B16/F10 melanoma cells compared to wild-type littermate controls. In addition, on generation of a primary subcutaneous tumor, metastasis to regional lymph nodes was clearly reduced in $T h y-1^{-/-}$mice. However, Thy-1 deletion did not affect subcutaneous primary tumor growth, tumor-induced recruitment of inflammatory cells or T cells, angiogenesis, or T-cell activation. In conclusion, Thy-1 contributes to metastasis of melanoma cells by mechanisms likely involving a Thy-1-mediated adhesion of melanoma cells to EC. (Am J Pathol 2013, 182: 266-276; http://dx.doi.org/10.1016/j.ajpath.2012.10.003)
\end{abstract}

The entry of tumor cells to the vascular or lymphatic circulation is a critical step during metastasis. ${ }^{1,2}$ Tumor cells use similar adhesion molecules for extravasation, which also play a role during recruitment of leukocytes to sites of inflammation. For example, $\alpha 4$ integrin expressed by melanoma cells mediates adhesion of melanoma cells to lymphatic vessels by interaction with vascular cell adhesion molecule-1 (VCAM-1). ${ }^{3}$

The $\alpha \mathrm{v}$ subunit is widely expressed in melanoma regardless of disease stage, whereas the $\beta 3$ expression on melanoma cells correlates with a poor clinical course. ${ }^{4}$ In addition to other functions of $\alpha v \beta 3$, this integrin mediates the heterophilic binding to CD31 (platelet endothelial cell adhesion molecule-1 [PECAM-1]) and L1, two cell adhesion molecules. ${ }^{4}$ Blocking $\alpha \mathrm{v} \beta 3$ or L1 partially inhibited transmigration of melanoma cells through an endothelial cell (EC) layer. ${ }^{5}$

A less-studied molecule in the context of tumor progression and metastasis is the adhesion molecule Thy-1 (CD90). Thy-1 is a highly glycosylated, GPI-anchored surface protein of the immunoglobulin superfamily. ${ }^{6,7}$ It is expressed on EC at sites of inflammation or in tumors, whereas EC in healthy tissue do not express Thy-1..$^{8-11}$ Originally, we identified human Thy-1 as an activation-associated cell adhesion molecule that mediates adhesion of leukocytes to activated microvascular EC via interaction with the $\beta 2$-leukocyte integrin Mac-1. ${ }^{11}$ In addition, we demonstrated in vitro that Thy-1 also mediates the adhesion of melanoma cells to activated EC via the interaction with the $\alpha v \beta 3$ integrin on melanoma cells. ${ }^{9,12}$ This interaction also plays a role in the communication of neurons and astrocytes. ${ }^{13,14}$

However, there are no available data about a possible role of Thy-1 in tumor progression and metastasis in vivo. Here, we demonstrated that Thy-1 is expressed on vascular and lymphatic EC in human melanoma, whereas in healthy skin and nevi Thy-1 was not expressed on vessels. VEGF and tumor necrosis factor- $\alpha$ (TNF- $\alpha$ ) were identified as inducers of Thy-1 expression on EC. The physiological role of Thy-1 in tumor metastasis was confirmed in metastasis models using Thy $-1^{-/}$mice. Taken together, tumor cells use Thy-1 on EC for adhesion to facilitate their metastatic behavior.

Supported by grants from the Deutsche Forschungsgemeinschaft (SA 683/2-1 to A.S. and U.A.) and the Europäischer Sozialfond (U.A.). 


\section{Materials and Methods}

\section{Materials}

Anti-human CD31-APC, anti-human CD90-PE from Miltenyi Biotec (Bergisch Gladbach, Germany), and antihuman podoplanin from Cell Sciences (Canton, MA) were used for flow cytometry. For immunofluorescence staining of human tissues, the following antibodies were purchased: anti-human VEGF and anti-human LYVE-1 from R\&D (Wiesbaden, Germany); anti-human TNF- $\alpha$ from Abcam, anti-human CD31 from eBioscience (San Diego, CA); antihuman CD90-PE from Miltenyi. Anti-mouse LYVE-1 from Cell Sciences, anti-mouse CD31 from eBioscience, antimouse Meca 32 from Pharmingen (Heidelberg, Germany), anti-mouse von Willebrand factor from Millipore (Schwalbach, Germany), and anti-mouse Thy-1-PE, anti-mouse CD11b-PE, anti-mouse CD3e-PE, and anti-mouse CD4-PE from Miltenyi were used for immunofluorescence staining of murine tissues. Secondary antibodies (anti-mouse-Alexa 488, anti-goat-Alexa 546, anti-goat-Alexa 488, antirat-Alexa 488, anti-rabbit-Alexa 488) were purchased from Invitrogen (Darmstadt, Germany). Phorbol12-Myristate-13-Acetate (PMA) was obtained from SigmaAldrich (Deisenhofen, Germany). TNF- $\alpha$, interleukin (IL) $-1 \beta$, IL6, IL8, VEGF-A ${ }_{165}$, stromal derived factor, and PDGF AB were purchased from Miltenyi.

\section{Cell Culture of Human Dermal Microvascular EC}

Epidermis was removed from human foreskin after dispase treatment (2.4 U/mL; Roche Diagnostics, Mannheim, Germany) overnight at $4^{\circ} \mathrm{C}$. The dermis was incubated for 30 minutes at $37^{\circ} \mathrm{C}$ with collagenase $(0.25 \%$ in HEPES $/ 2 \mathrm{mmol} /$ $\mathrm{L} \mathrm{CaCl}_{2}$; Sigma-Aldrich). EC were carefully scraped out of the dermis and cultured in EC medium with $2 \%$ fetal calf serum (Promocell, Heidelberg, Germany). Human dermal microvascular endothelial cell (HDMEC) cultures were characterized by their typical cobblestone morphology using light microscopy and expression of CD31 by flow cytometry.

B16/F10 melanoma cells were grown in Dulbecco's modified Eagle's medium (DMEM; Biochrom, Berlin, Germany) supplemented with $10 \%$ fetal calf serum and $1 \%$ penicillin/streptomycin.

Chinese hamster ovary (CHO) cells stably transfected with murine Thy-1 (mThy-1, full-length Thy-1 cDNA; accession no. MM_009382 resulting in the complete mature protein) or vector alone were cultured in DMEM with $10 \%$ fetal calf serum, $1 \%$ penicillin/streptomycin, and $7.5 \mu \mathrm{g} / \mathrm{mL}$ blasticidin (Invitrogen).

\section{Adhesion Assay}

B16/F10 melanoma cells were labeled using a carboxyfluorescein diacetate cell tracker kit (Invitrogen) according to the manufacturer's instructions. Labeled cells $\left(0.5 \times 10^{5}\right)$ were added to a confluent monolayer of vector-transfected $\mathrm{CHO}$ cells or mThy-1-transfected $\mathrm{CHO}$ for 15 minutes. Unbound cells were removed by several washes with phosphate-buffered saline (PBS). The number of adherent cells was calculated from 10 independent photographs per sample using BZ-9000E analyzer software version 1.42 (Keyence, Neu Isenburg, Germany).

\section{Activation and Proliferation of T Cells}

Murine $\mathrm{CD}^{+} \mathrm{T}$ cells were isolated from the spleen using a Pan T Cell Isolation Kit according to the original protocol (Miltenyi). CD ${ }^{+} \mathrm{T}$ cells $\left(1 \times 10^{5}\right)$ were stimulated with antiCD3/CD28 magnetic beads (Invitrogen) in a cell-to-bead ratio of 1:2. For analysis of proliferation, $T$ cells were labeled with carboxyfluorescein diacetate (Invitrogen), and stimulation was performed in the presence of $100 \mathrm{U} / \mathrm{mL}$ of IL2 (Biolegend, San Diego, CA). Proliferation was measured after 4 days, and expression of CD25 was detected after 24 hours by flow cytometry on gating on $\mathrm{CD} 4^{+}$cells.

\section{Flow Cytometry}

Endothelial cells $\left(2 \times 10^{5}\right)$ were incubated for 1 hour with $0.5 \mu \mathrm{g}$ of anti-podoplanin antibody and subsequently with an anti-rabbit-Alexa 488 antibody (1:500). Finally, cells were incubated with anti-human CD31-APC for detection of EC and with anti-human CD90-PE for analysis of Thy-1 expression. After washing with PBS/10\% Gelafusal (Bernburg, Germany), cells were analyzed by flow cytometry (FC 500; Beckman Coulter, Krefeld, Germany).

\section{Immunofluorescence Staining}

Cryostat sections of five donors of healthy skin, five patients with nodular or melanocytic nevi, five patients with nodular melanoma, and five patients with subcutaneous melanoma metastases were stained with primary antibodies or isotype control antibody diluted in PBS/0.1\% Tween 20/3\% bovine serum albumin, and subsequently with the respective secondary, labeled antibody. In double staining procedures, incubation with a directly labeled antibody followed. Cell nuclei were stained with DAPI. All washings were performed with $\mathrm{PBS} / 0.1 \%$ Tween 20. Immunofluorescence images were photographed with a BZ-9000E microscope (Keyence). The number of labeled cells was calculated using BZ-9000E analyzer software version 1.42 (Keyence).

\section{RNA Preparation and PCR}

Tissue samples were solubilized using TRIsure (Bioline, Luckenwalde, Germany), and RNA was isolated by phenol/ chloroform extraction. A $0.5-\mu \mathrm{g}$ quantity of total RNA was used for first-strand cDNA synthesis with M-MLV Reverse Transcriptase (Promega, Madison, WI) according to the manufacturer's protocol. The following primers (Metabion, 
Martinsried, Germany) were used for PCR: RS36B4Forward: 5'-GGACCCGAGAAGACCTCCTT-3'; RS36B4Reverse: 5'-GCACATCACTCAGAATTTCAATGG-3'; Pmel17-Forward: 5'-CGGATGGTCAGGTTATCTGG-3'; Pmel17-Reverse: 5'-ATGGTGAAGGTTGAACTGGC-3' PCR products were separated in a $1.5 \%$ agarose gel.

\section{Mice}

Thy $-1^{-1-}$ mice were a kind gift from Prof. Roger Morris (King's College London). Thy-1-deficient (Thy-1 ${ }^{-/-}$) mice had been established on a $129 / \mathrm{Sv} \times \mathrm{C} 57 \mathrm{BL} / 6$ background as described previously. ${ }^{15}$ Here, the mice were back-crossed on C57/BL6 background six times. F2 littermates from the intercross of F1 Thy $-^{+/-}$mice were used for comparative

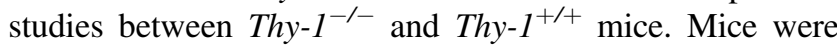
provided with food and water ad libitum and were kept under a 12-hour light-dark cycle. All animal experiments were performed according to institutional and state guidelines. The Committee on Animal Welfare of Saxony approved animal protocols used in this study (TVV31/09).

\section{Experimental Lung Metastasis Model}

A total of $5 \times 10^{5}$ B16/F10 cells ( $>90 \%$ viability) in $100 \mu \mathrm{L}$ of PBS were injected into the lateral tail vein of 8- to 12-week-old Thy-1 ${ }^{-1-}$ mice and wild-type littermates. At day 7 , mice were sacrificed and lungs were prepared. Tumor nodules were counted, and images of the lungs were taken in a standardized fashion with a stereo microscope (Motic SMZ-168-BL, Motic Group Co., Xiamen, China). Lungs were frozen for preparation of cryosections.

\section{Subcutaneous B16/F10 Melanoma Model}

B16/F10 melanoma cells $\left(1.5 \times 10^{6}\right)$ in $100 \mu \mathrm{L}$ of PBS were injected subcutaneously into the back flank of Thy $-1^{-/-}$mice or wild-type littermates. After indicated time points, mice were sacrificed. Tumor weight was determined, and tumor tissue was frozen for preparation of tissue sections. Inguinal lymph nodes were treated with TRISure (Bioline) to isolate RNA.

\section{Lung Inflammation}

Mice were immunized with ovalbumin by a standard immunization protocol as described elsewhere. ${ }^{10}$

\section{Statistical Analysis}

Depending on the normality of the data, analysis was performed using the Mann-Whitney rank sum test or Student's $t$-test. For comparison of more than two groups, one-way analysis of variance was used. Values of $P<0.05$ were considered to be significant. For testing the significance of the rate of tumor metastasis, the Fisher exact test was used.

\section{Results}

\section{Thy-1 Is Highly Expressed on EC in Melanoma}

Recently, we identified Thy-1 as a cell adhesion molecule on activated human microvascular EC at sites of inflammation. $^{11,12}$ Here, we report that Thy-1 is also highly expressed on EC in melanoma. EC-associated Thy-1 expression was detected by immunofluorescence co-staining of Thy-1 and CD31 as EC marker. In healthy skin (Figure 1, A-D) and nevi (Figure 1, F-I) Thy-1 was not expressed on EC. In contrast, in human melanoma (Figure $1, \mathrm{~K}-\mathrm{N}$ ) and cutaneous melanoma metastases (Figure $1, \mathrm{P}-\mathrm{S}$ ), strong Thy1 expression was detected on EC. Negative controls without primary antibody did not show any signal (Figure 1, E, J, O, and T). H\&E staining demonstrates the morphology of the tissue samples (Figure 1, U-X). By double immunostaining of Thy-1 and LYVE-1 (Figure 1Y), the expression of Thy-1 on both lymphatic (Lyve- $1^{+} /$Thy $-1^{+}$; arrow) and blood vessels (Lyve- $1^{-} /$Thy- $1^{+}$; asterisk) was demonstrated.

To identify potential inducers of Thy-1 expression on EC, HDMEC were stimulated with growth factors and cytokines known to play a role in tumor progression. Subsequently, Thy-1 expression on CD31-positive EC was measured by flow cytometry. PMA was used as positive control. As shown in Figure 2A, PMA, TNF- $\alpha$, and VEGF induced Thy-1 expression on HDMEC, whereas IL-1 $\beta$, IL-6, IL-8, stromal derived factor, and platelet derived growth factor $\mathrm{AB}$ did not. A time-dependent analysis revealed a fast induction of Thy- 1 after 14 hours, which peaked after 3 to 4 days on stimulation with VEGF (Figure 2B). Characterization of EC revealed that both lymphatic and blood vessel EC express Thy-1 on stimulation with PMA, TNF- $\alpha$, and VEGF, respectively (Figure 2C). However, lymphatic EC displayed stronger Thy-1 expression on stimulation. To underline the physiological role of VEGF and TNF- $\alpha$ in Thy-1 stimulation in melanoma, we analyzed the expression of VEGF/TNF- $\alpha$ and Thy- 1 in human melanoma tissues. Indeed, by immunofluorescence staining, we observed a close colocalization of Thy-1 and VEGF or TNF$\alpha$ expression (Figure 2, F and G). Controls with isotype antibodies showed no staining (Figure 2, D and E).

Because Thy- 1 displays a species-specific expression pattern, ${ }^{16}$ we analyzed Thy-1 expression on EC in tumors of mice. In primary melanoma generated by subcutanous injection of B16/F10 melanoma cells, Thy-1 expression on vascular and lymphatic EC was analyzed by immunofluorescence co-staining of Thy-1 and CD31, Lyve-1, Meca32, respectively. As shown in Figure 3, Thy-1 was strongly expressed on EC as shown by co-staining of vessels with an anti-CD31 and an anti-Thy-1-antibody (Figure 3, A-C). Staining of lymphatics by an anti-Lyve-1 antibody revealed the expression of Thy-1 on lymphatic endothelial cells in melanoma (Figure 3, D-F). Moreover, inside the tumor, many Lyve-1-negative/Thy-1-positive cells were observed, suggesting that Thy-1 is massively expressed on 


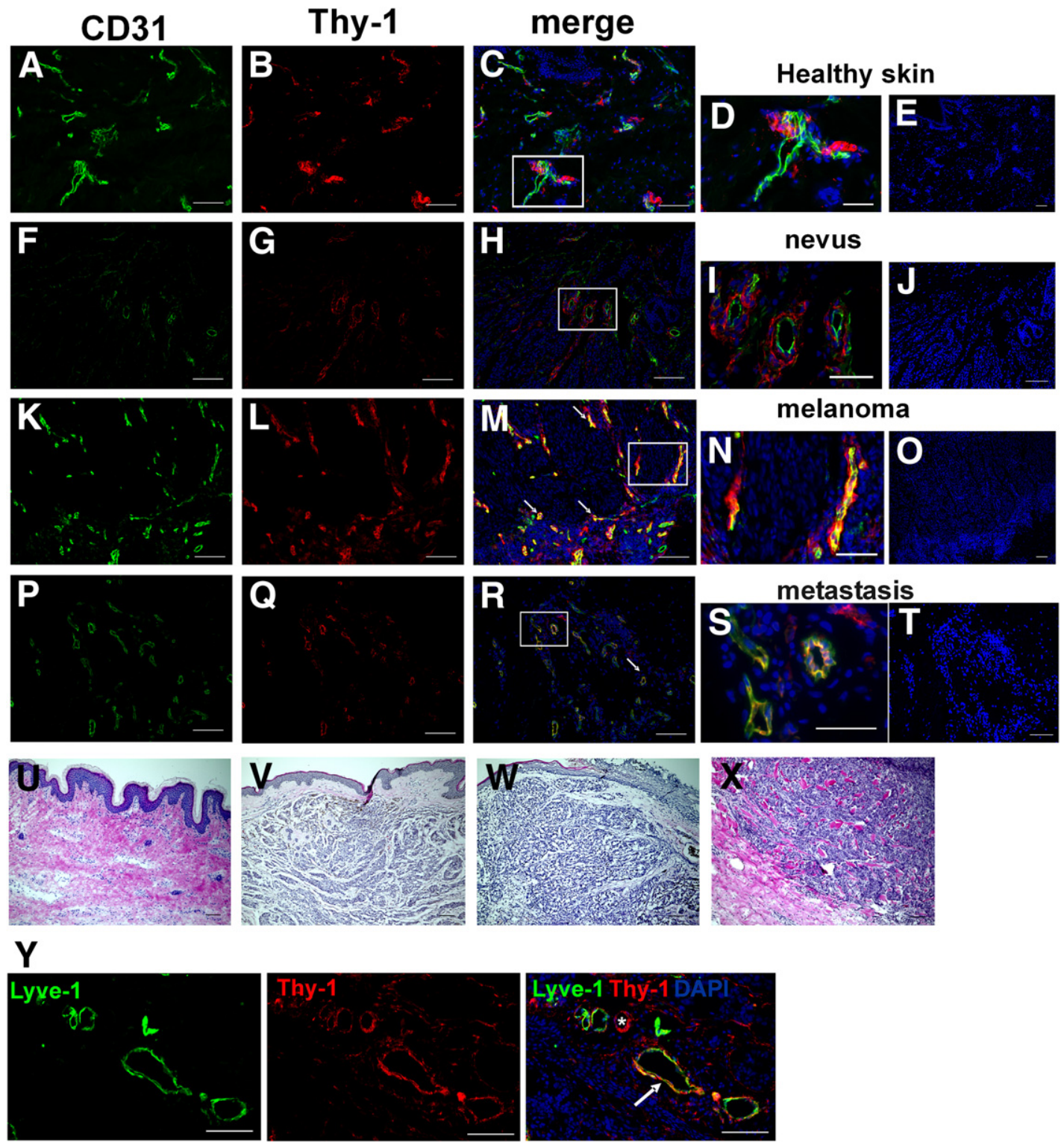

Figure 1 Human Thy-1 expression is induced on vascular and lymphatic EC in melanoma and cutaneous metastasis. Representative images of Thy-1 expression in healthy skin $(\mathbf{A}-\mathbf{D})$, nevus $(\mathbf{F}-\mathbf{I})$, melanoma $(\mathbf{K}-\mathbf{N})$, cutaneous melanoma metastases $(\mathbf{P}-\mathbf{S})$ detected by immunofluorescence costaining of Thy-1 (red) and the EC marker CD31 (green). E, J, 0, and $\mathbf{T}$ show staining with isotype control antibodies. H\&E stainings of the respective tissue samples are shown in $\mathbf{U}$ (healthy skin), $\mathbf{V}$ (nevus), $\mathbf{W}$ (melanoma), and $\mathbf{X}$ (cutaneous melanoma metastasis). Y: Double immunostaining of the lymphatic marker LYVE-1 (green) and Thy-1 (red). Thy-1 is expressed on blood vessel (LYVE-1 ${ }^{-}$, Thy $-1^{+}$; asterisk) and lymphatic vessel (yellow) by colocalization of LYVE-1 and Thy-1 (arrow). Cell nuclei were stained with DAPI (blue). Each image shows one representative example from five patients. Scale bar $=100 \mu \mathrm{m}$.

blood vessels. Double staining of Thy-1 and Meca32 (Figure 3, G-I), which detect vascular EC, ${ }^{17}$ confirmed that Thy-1 is expressed on vascular EC in melanoma. Control with isotype antibodies showed no staining (Figure 3J). An overview of the tissue sample is provided by $\mathrm{H} \& \mathrm{E}$ staining (Figure $3 \mathrm{~K}$ ). The results were demonstrated by staining of Thy-1 on EC, lypmphatics, and blood vessel EC in lungs on induction of tumor metastasis after i.v. injection of B16/F10 melanoma cells (data not shown). Taken together, Thy-1 is expressed on both blood vessel and lymphatic EC in melanoma in humans and in mice.
Metastasis Formation Is Diminished in Thy-1-Deficient Mice

Because of the presence of Thy- 1 on both vascular and lymphatic EC, the role of Thy- 1 for hematogenous and lymphogenous metastasis was studied.

The functional importance of Thy- 1 for hematogenous tumor cell metastasis was assessed on development of pulmonary metastases after tail vein injection of B16/F10 melanoma cells into Thy-1 $1^{-/}$mice and wild-type littermate C57BL/6 controls. In Thy-1-deficient mice, the number of 
A

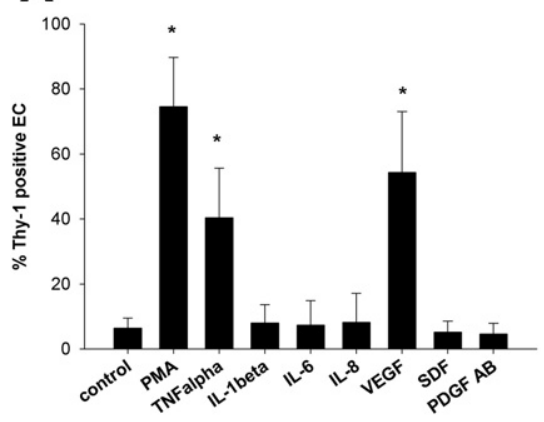

C
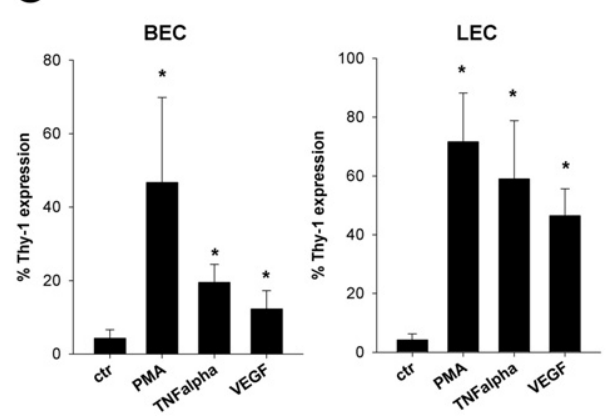

B
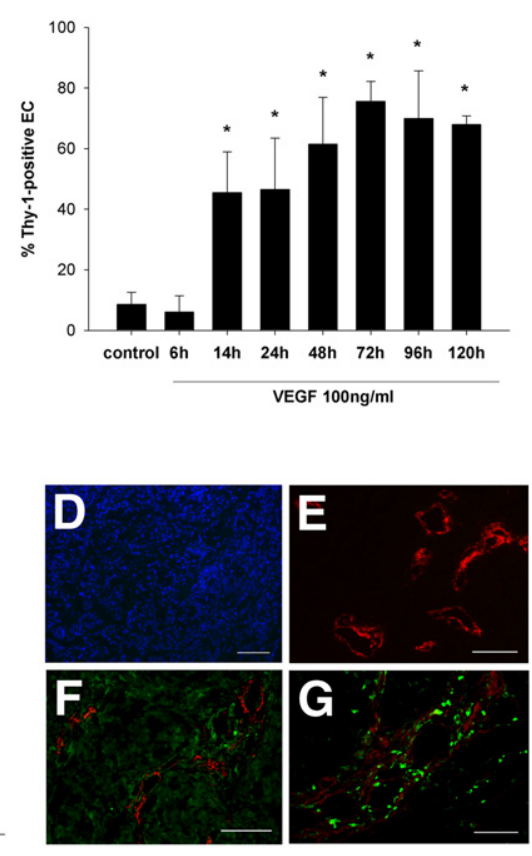

Figure 2 VEGF and TNF- $\alpha$ induce Thy-1 expression on human dermal microvascular endothelial cells (HDMEC). HDMEC were stimulated as indicated. Thy-1 expression on CD31-positive EC was measured by flow cytometry. A: HDMEC were stimulated for 48 hours with $20 \mathrm{ng} / \mathrm{mL}$ of PMA, TNF- $\alpha$, IL-1 $\beta$, or $100 \mathrm{ng} / \mathrm{mL}$ of VEGF, IL-6, IL-8, stromal derived factor, or platelet derived growth factor $A B$, respectively. B: HDMECs were stimulated with $100 \mathrm{ng} / \mathrm{mL}$ of VEGF for 6 to 120 hours. C: HDMECs were stimulated for 48 hours with 20 $\mathrm{ng} / \mathrm{mL}$ PMA, $20 \mathrm{ng} / \mathrm{mL}$ TNF- $\alpha$, and $100 \mathrm{ng} / \mathrm{mL}$ VEGF, respectively. Thy-1 expression on $\mathrm{CD} 31^{+} /$ podoplanin ${ }^{+}$cells [lymphatic endothelial cells (LEC)] and on $\mathrm{CD}^{+} 1^{+} /$podoplanin ${ }^{-}$cells [blood vessel endothelial cell (BEC)] was detected after 48 hours by flow cytometry. ${ }^{*} P<0.01(n=5)$ compared to control without stimulation. $\mathbf{D}-\mathbf{G}$ : Detection of TNF- $\alpha(\mathbf{F})$ and VEGF (G; green) in close co-localization to Thy-1 (red) in human melanoma by immunofluorescence staining. Controls were stained without any primary antibody (D) or without VEGF or TNF- $\alpha$ antibody (E). One representative example of three is shown. Scale bar $=$ $100 \mu \mathrm{m}$. lung metastases was significantly diminished compared to that in controls (Figure 4, A and B). Detailed microscopic analysis of lung tissue sections consistently revealed larger numbers and sizes of metastatic foci in lungs of wild-type mice (Figure 4, C and D).

To study the importance of Thy-1 for lymphogenous metastasis, B16/F10 melanoma cells were subcutaneously applied to $T h y-1^{-/-}$mice and wild-type littermates, inducing a subcutaneous melanoma. After 17 days, the inguinal lymph nodes were prepared, and the expression of the melanoma cell-specific gene (Pmel-17 ${ }^{18}$ ) was detected by reverse transcription (RT)-PCR. In untreated mice, Pmel-17 was not detectable in lymph nodes, blood, ovary, kidney, spleen, or liver (data not shown). On induction of subcutaneous melanoma in 10 of 19 wild-type mice, melanoma cells were detected in lymph nodes by RT-PCR of Pmel-17. In contrast, the presence of melanoma cells in the inguinal lymph nodes could be identified only in three of 15 Thy- $1^{-/-}$mice (Figure 4E). However, compared to that in Thy $-1^{+/+}$mice, this decrease did not reach statistical significance $(P=0.079)$.

Next, we looked at underlying mechanisms of the reduced metastasis formation in lung in Thy- $1^{-/-}$mice, focusing on density of microvessels, recruitment of immune cells, and activation of the immune system. Previously, we reported that Thy-1 acts as an adhesion molecule for the interaction of melanoma cells to microvascular EC via the binding to $\alpha \mathrm{v} \beta 3$ integrin. $^{12}$ Adhesion and subsequent transmigration of melanoma cells to activated microvascular EC was significantly inhibited after blocking Thy-1 on EC or $\alpha v \beta 3$ on melanoma cells. ${ }^{12}$ Here, we confirmed this data showing a strong, Thy-1-dependent adhesion of murine B16/F10 melanoma cells to $\mathrm{CHO}$ cells transfected with murine Thy-1 in vitro (Figure 5A). In vivo, we found similar microvascular densities in the experimental tumors in Thy $1^{+/+}$and Thy $-1^{-/-}$mice, detected by staining of vessels by von Willebrand factor (Figure 5B). Furthermore, we did not detect any significant infiltration of the lung with myeloid cells in either Thy $-1^{+/+}$or Thy-1 $1^{-/-}$mice as determined by immunofluorescence staining of $\mathrm{CD} 11 \mathrm{~b}^{+}$cells. In contrast, high numbers of $\mathrm{CD} 1 \mathrm{~b}^{+}$myeloid cells were observed on induction of lung inflammation by ovalbumin challenge in a control experiment (Figure 5C). Because of the increased number of metastatic nodules in the lung, we analyzed whether Thy-1 provided a pro-proliferative microenvironment. Primary melanoma was induced by subcutaneous application of B16/ F10 melanoma cells into the dorsal flanks of Thy $-1^{-/-}$mice and littermate controls. Tumor weights at 7, 12, and 17 days were indistinguishable, indicating that Thy-1 itself did not support melanoma cell proliferation and tumor growth (Figure 5D).

Because Thy- 1 is highly expressed on T cells, we excluded that $\mathrm{T}$-cell functions are affected in $T h y-1^{-1-}$ mice. The number of $\mathrm{CD}^{+} \mathrm{T}$ cells in the spleen was similar in Thy-1 $1^{-/-}$ mice and littermate controls (Figure 5E). Furthermore, we looked at the infiltration of the lung with $\mathrm{T}$ cells on induction of metastasis formation, and found no differences between Thy $-1^{+/+}$and $T h y-1^{-1-}$ mice (Figure 5F). Finally, we analyzed the expression of CD25 and proliferation of T cells from $T h y-1^{+/+}$and Thy-1 $1^{-/-}$mice on activation with antiCD3/CD28 to exclude functional defects. As shown in Figure $5 \mathrm{G}$, there were no differences in the proliferation of $\mathrm{T}$ cells from $T h y-1^{-/-}$mice and littermate controls on 

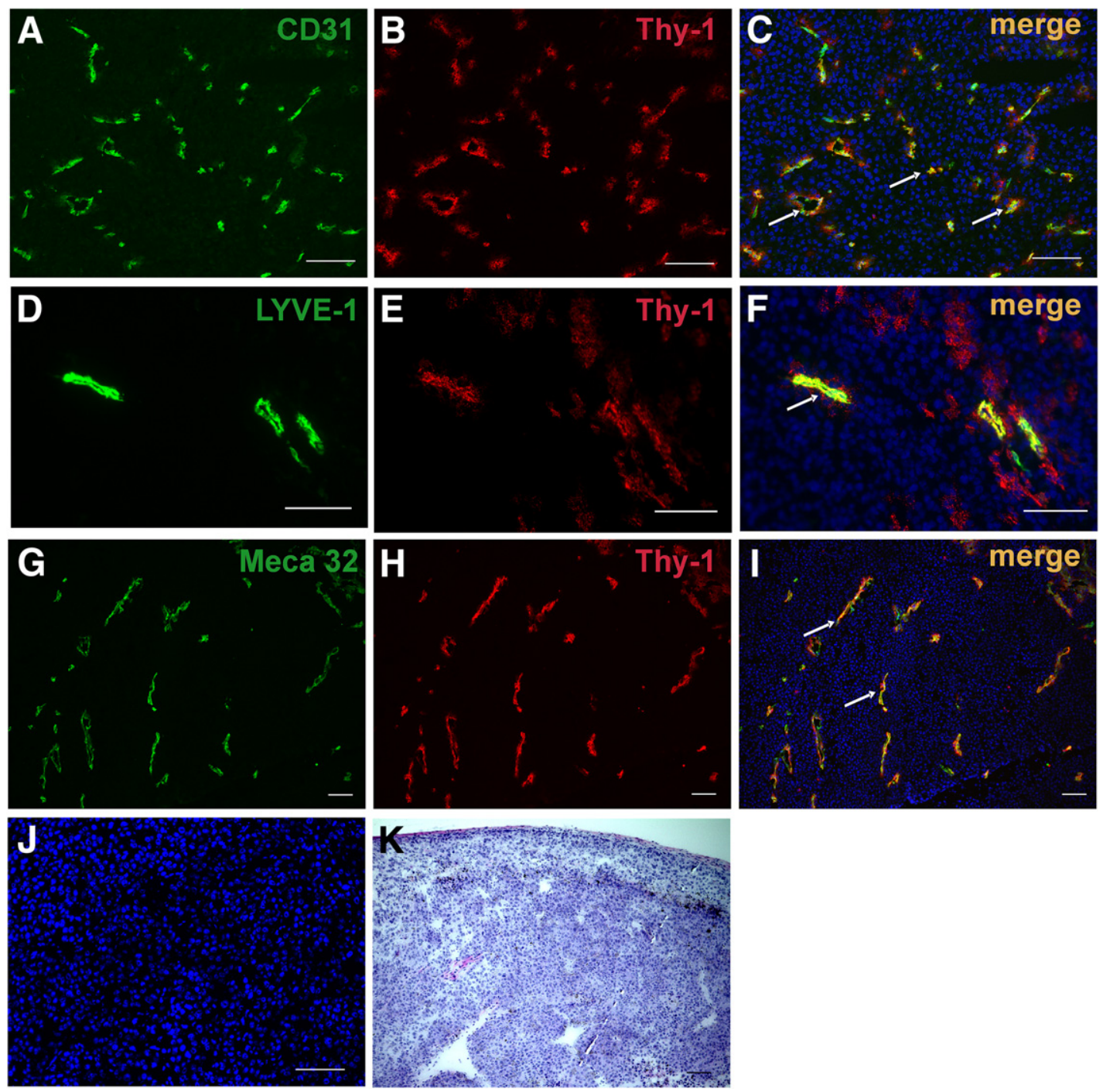

Figure 3 Murine Thy-1 is expressed on blood vessels and lymphatic EC in melanoma. Subcutaneous melanoma was generated by s.c. injection of B16/F10 melanoma cells in mice. Representative images show the co-immunostaining of Thy-1 (red) and CD31 (A-C), Lyve-1 (D-F), and Meca32 (G-I), respectively. J: Staining with isotype control antibody. K: H\&E staining of the respective tissue sample. Co-localization of Thy-1 with the pan-endothelial cell marker CD31, the lymphatic endothelial cell marker LYVE-1, and blood vessel marker Meca32 (yellow, arrows) indicates the expression of Thy-1 on blood vessels and lymphatic EC in melanoma in mice. Scale bar $=100 \mu \mathrm{m}$.

stimulation with a CD3/CD28 in the presence of IL-2. In accordance, $\mathrm{T}$ cells from $T h y-1^{-/-}$mice did not display a defect in the expression of CD25 on stimulation (Figure 5H). In summary, the lack of Thy- 1 on T cells neither affected the immigration of $\mathrm{T}$ cells into the lung on metastasis formation nor reduced the activation and proliferation of $\mathrm{T}$ cells.

Thus, the reduced metastasis of melanoma cells in Thy $-1^{-1-}$ mice is most likely due to the lack of the Thy-1-mediated adhesion of melanoma cells to ECs.

\section{Discussion}

The metastatic potential of tumor cells is the risk-determining factor of many malignant diseases and results in poor prognoses. ${ }^{19,20}$ Adhesive interactions between tumor cells and the endothelium are critical steps during tumor metastasis. Mechanisms and pathways used by tumor cells are closely related to those used in physiological leukocyte migration and their invasion into peripheral tissues. Previously, we observed that human Thy-1 mediates the adhesion of myeloid cells to EC via the interaction with the integrin Mac-1 (CD11b/CD18) and is involved in the control of the extravasation of myeloid cells during inflammation in vivo. ${ }^{10,11}$ Moreover, Thy-1 on EC is a counterreceptor for av $\beta 3$ on melanoma cells and mediates the adhesion of melanoma cells to activated EC in vitro. ${ }^{12}$ Therefore, we analyzed the impact of Thy- 1 on melanoma metastasis in vivo.

In healthy skin and in benign melanocytic skin lesions (nevi), Thy-1 was not detectable on EC. In contrast, in 
A
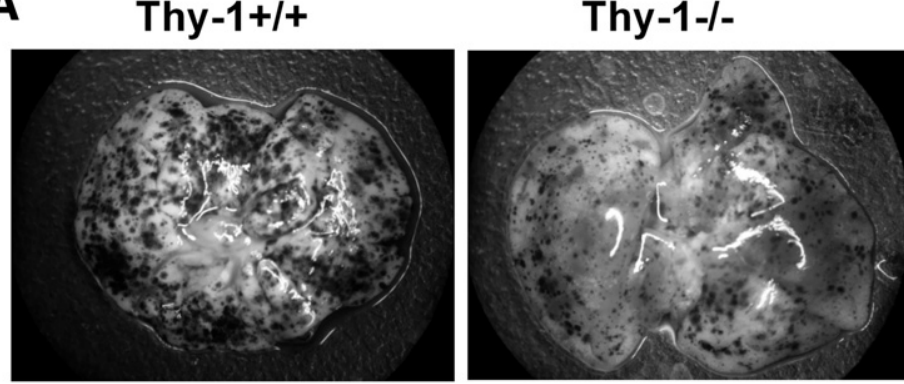

C

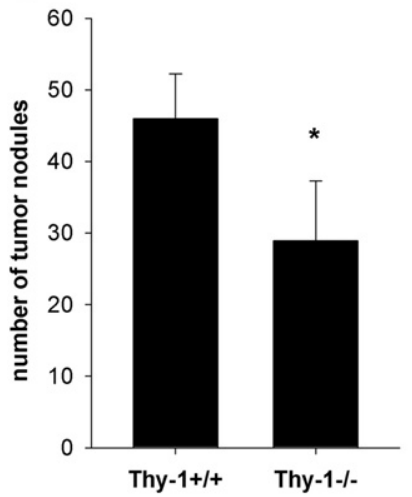

D

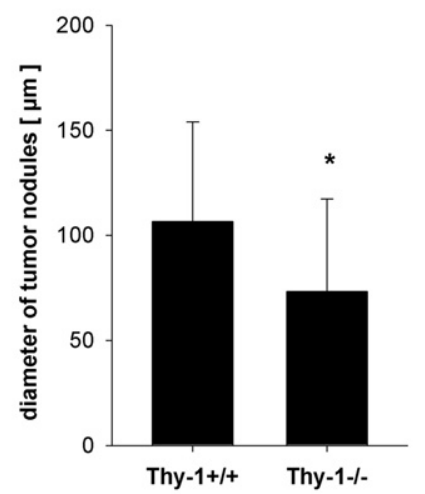

B

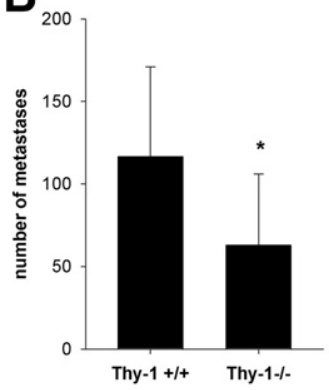

$\mathbf{E}$

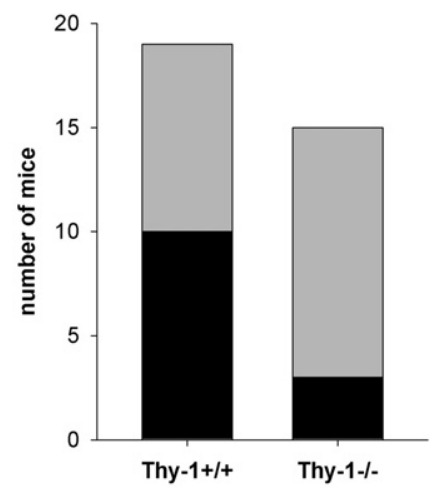

Figure 4 Lack of Thy-1 reduced pulmonary metastasis. Lung metastasis formation was generated by tail vein injection of B16/F10 melanoma cells into Thy- $1^{-/-}$mice and littermate wild-type $\mathrm{C57BL} / 6$. After 7 days, lungs were analyzed. A: Representative images of pulmonary metastatic foci 7 days after i.v. injection of melanoma cells. B: Number of experimental pulmonary metastatic nodules $(n=23)$. Number (C) and size (D) of metastatic nodules were determined in histological sections $\left(n=11 ;{ }^{*} P<0.05\right)$. E: B16/F10 melanoma cells were injected subcutaneously into the flank of wild-type C57/BL6 littermate controls and Thy-1 ${ }^{-/-}$C57/BL6 mice. After 17 days, evidence of lymph node metastases in inguinal lymph nodes was determined in 19 Thy-1 $1^{+/+}$and 15 Thy $-1^{-/-}$mice by detection of Pmel-17 by RT-PCR. Black indicates the number of mice with detection of Pmel17 in inguinal lymph nodes; gray indicates the number of mice with no detection of Pmel17 in inguinal lymph nodes.

primary melanoma as well as in subcutaneous metastases, Thy-1 was clearly detectable on EC identified by CD31 staining. Previously, we showed the induction of Thy- 1 on microvascular EC by soluble mediators of human melanoma cell lines. ${ }^{12}$ Here, we identified VEGF-A and TNF- $\alpha$ as potent inducers of Thy-1 expression on microvascular EC. TNF- $\alpha$ is expressed by both leukocytes and tumor cells in melanoma. ${ }^{21,22}$ VEGF can be produced by many types of cells including fibroblasts, macrophages, neutrophils, EC, or tumor cells on stimulation by hypoxia or cytokines. ${ }^{23,24}$ Because we observed Thy- 1 in close co-localization to TNF- $\alpha$ and VEGF in human melanoma, we suppose that VEGF and TNF- $\alpha$ might be inducers of the Thy-1 induction on EC in melanoma. There are several data showing a significantly weaker expression of VEGF in nevi compared to melanoma (Konstantina et al, 2011; Mehnert et al, 2010). Thus, we assume that the different VEGF expression in melanoma and nevi might be one responsible factor for the different expression of Thy-1 in nevi and melanoma. Double staining of Thy- 1 and VEGF in melanoma revealed a close co-localization, suggesting that VEGF might indeed be responsible for high expression of Thy-1 on EC in melanoma.
Tumor cells use two principal pathways of metastasis: the lymphogenic metastasis to regional lymph nodes, and hematogenic metastasis to distant organs. ${ }^{25}$ To realize the importance of Thy- 1 for both pathways, the expression of Thy-1 on lymphatic and blood vessel endothelial cells was determined. Jurisic et $\mathrm{al}^{26}$ described Thy- 1 as a marker for lymphatic vessels. ${ }^{26}$ Ex vivo isolated murine lymphatic endothelial cells (LEC) from murine colon tissue expressed much more Thy-1 than blood vessel endothelial cells (BEC). In contrast to several other groups,${ }^{27-31}$ these investigators did not find an upregulation of Thy-1 on EC during inflammation. Nevertheless, in human prostate cancer, podoplanin-positive LEC as well as several podoplanin-negative EC, representing blood vessels, revealed a clear Thy- 1 expression. ${ }^{26}$ In the present study, both Lyve-1-positive LEC and Lyve-1-negative BEC showed clear Thy-1 expression in human melanoma. Moreover, stimulation of human dermal microvascular EC with PMA, TNF- $\alpha$, and VEGF induced Thy-1 expression on both BEC $\left(\mathrm{CD} 31^{+} /\right.$podoplanin $\left.^{-}\right)$and LEC $\left(\mathrm{CD} 31^{+} /\right.$ podoplanin $\left.{ }^{+}\right)$. Although Thy-1 was strongly expressed on both LEC and BEC in situ, activated cultured BEC expressed lower levels of Thy-1 than activated cultured 


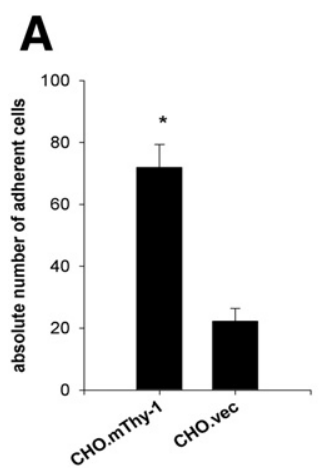

D

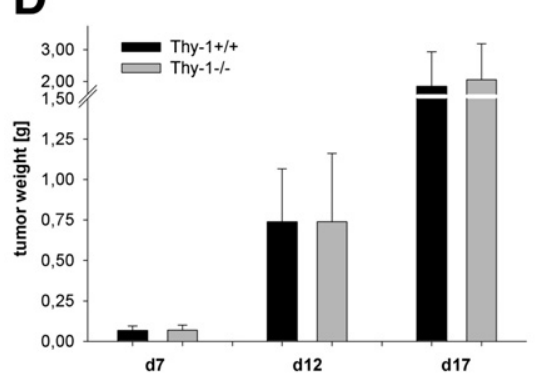

B

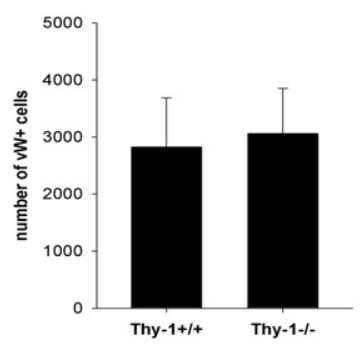

E

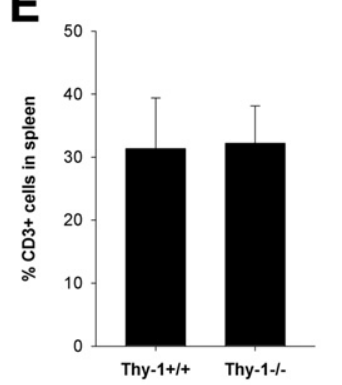

C

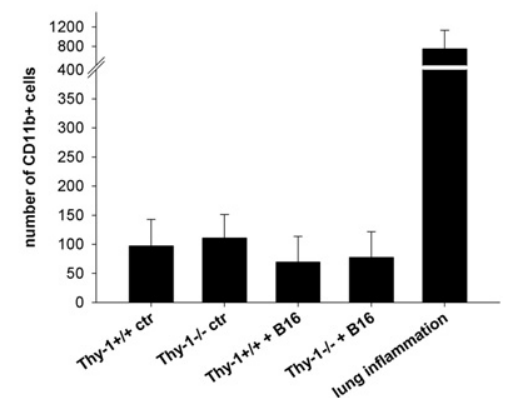

$F$

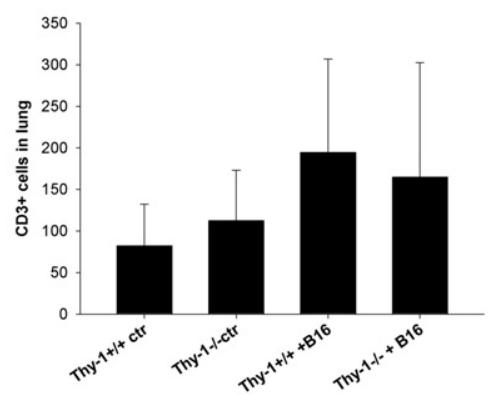

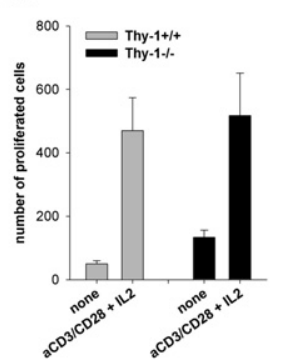

H

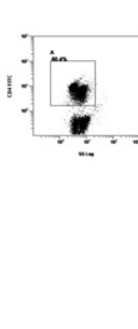

Thy-1+/+

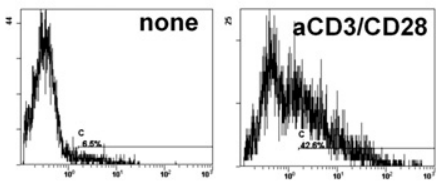

Thy-1-/-

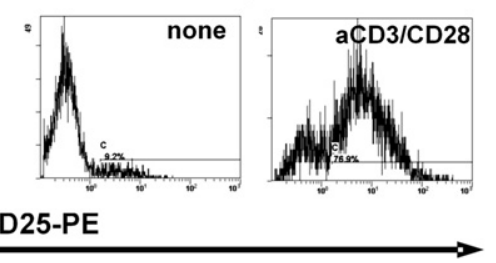

Figure 5 Mechanisms of diminished metastasis in Thy-1 $1^{-/-}$mice. A: Adhesion of B16/F10 melanoma cells to CH0 cells transfected with murine Thy-1 (CH0.mThy-1) and vector-transfected control cells (CH0.vec). Tissue sections were photographed using a Keyence BZ-9000E microscope, and the number of positive cells was counted using Keyence BZ-9000E analyzer software. Data represent mean \pm SD of three experiments ( $P<0.05$ ). B: Lung metastasis formation was generated by tail vein injection of B16/F10 melanoma cells into Thy-1 ${ }^{-/-}$mice and wild-type littermate C57BL/6. Microvascular density was detected after 7 days by staining of vessels with anti-von Willebrand factor $\left(\mathrm{vW}^{+}\right)(n=11)$ and quantified using Keyence BZ-9000E analyzer software. Data represent mean \pm SD of three experiments ( $P<0.05$ ). C: Infiltration of myeloid cells was determined by staining with an anti-CD11b antibody after 7 days. In addition, the lungs of untreated Thy $-1^{-/-}$and $T h y-1^{+/+}$mice were analyzed. Infiltration of lung with inflammatory cells in an experimentally induced lung inflammation model was used as a positive control ${ }^{10}(n=12)$. D: B16/F10 melanoma cells were injected subcutaneously into dorsal flanks of Thy- ${ }^{-/-}$mice and littermate controls. After 7, 12, and 17 days, tumor weight was determined $(n=5)$. E: The content of T cells in spleen in Thy-1 ${ }^{+/+}$and Thy-1 ${ }^{-/-}$mice was detected by flow cytometry. Dead cells were excluded by propidium iodide staining $(n=3)$. F: B16/F10 melanoma cells were applied intravenously into Thy $-1^{-/-}$mice and wild-type littermate $\mathrm{C} 57 \mathrm{BL} / 6$. As control, the lungs of untreated Thy-1 $1^{-/-}$and Thy-1 $1^{+/+}$mice were used. Infiltration of CD3 ${ }^{+}$T cells was determined by staining with an anti-CD3 antibody $(n=12)$. G: T cells from Thy-1 ${ }^{-/-}$mice and littermate controls were labeled with carboxyfluorescein diacetate and activated by anti-CD3/CD28 and IL-2. After 4 days, proliferation of CD4 $^{+}$cells was detected by flow cytometry. Control cells were cultured in medium alone $(n=3)$. H: T cells from Thy $1^{-/-}$mice and littermate controls were activated by anti-CD3/CD28. For analysis, cells were gated on forward/side scatter. Dead cells were excluded by propidium iodide staining. CD25 expression on CD4 ${ }^{+} \mathrm{T}$ cells was measured by flow cytometry after 24 hours. Histogram shows one representative example of three.

LEC. This might be due to the stability of the cell specific gene expression pattern in cultured cells. Amatschek et al ${ }^{32}$ described the strict control of EC specific genes by the in vivo tissue environment. To investigate the impact of Thy-1 expression on both BEC and LEC for tumor metastasis, we first extended the analysis of Thy-1 expression to an experimentally induced, subcutaneous murine melanoma model and a metastatic lung model. Double staining with
CD31 and Meca32 or LYVE-1 clearly confirmed a high expression of Thy-1 on both BEC and LEC in murine melanoma as well.

Taking these findings together, because of the high expression of Thy-1 on both BEC and LEC in melanoma, it appears that Thy-1 might be involved in the lymphogenic as well as in the hematogenic metastasis during melanoma progression. 
Using the B16/F10 lung metastasis model in Thy-1 $1^{-/}$ mice and wildtype littermates as a model for hematogenic metastasis, we demonstrated an essential role of Thy- 1 for hematogenic metastasis in melanoma. Indeed, both the number and size of tumor nodules were significantly diminished in $T h y-1^{-/}$mice.

The role of adhesion molecules for the translymphatic migration is controversially discussed. The process of metastasis is closely related to the pathways and mechanisms used in physiological leukocyte migration. ${ }^{25}$ On the one hand, Pflicke et $\mathrm{al}^{33}$ showed that lymphatic migration of dendritic cells is independent of integrin-mediated adhesive interactions. In contrast, Johnson et $\mathrm{al}^{34,35}$ found that lymphatic endothelium undergoes a program of activation during inflammation, including upregulation of intracellular adhesion molecule-1 (ICAM-1) and vascular cell adhesion molecule-1 expression to support increased leukocyte transmigration. Consistent with findings by Johnson et al, ${ }^{36}$ our studies showed a massive upregulation of Thy-1 on LEC by the inflammatory mediator TNF- $\alpha$ as well as by the tumor environment. Moreover, in an oxazolone-induced skin hypersensitivity model, blocking of adhesion molecules impaired migration of resident skin Langerhans cells as well as exogenously added bone marrow-derived dendritic cells to the draining lymph nodes. Johnson et $\mathrm{al}^{35}$ concluded that, in resting LEC, CCL21 stimulates transendothelial migration by a predominantly chemotactic mechanism and in TNF- $\alpha$-stimulated LEC by a $\beta 2$ integrin-mediated mechanism. Rebhun et $\mathrm{al}^{3}$ demonstrated the importance of adhesion molecule ( $\alpha 4$ integrin)-mediated interactions for lymphogenic migration of melanoma cells supporting our findings. In the present study, on subcutaneous application of B16/F10 melanoma cells, a clear reduction of the incidence of melanoma cells in the draining lymph nodes was observed, proving a role of Thy- 1 in lymphogenic metastasis.

To obtain insight into the mechanisms of the reduced metastases in Thy $-1^{-1-}$ mice, the infiltration of inflammatory cells was analyzed, as we have shown the role of Thy-1 in the regulation of the extravasation of myeloid cells during inflammation. ${ }^{10}$ In accordance with van Spriel et al and Yamada et $\mathrm{al}^{37,38}$, we did not find an infiltration of myeloid cells into the lung on metastasis formation. Because we did not find any differences in the number of myeloid cells in Thy $-1^{-/-}$mice and controls, we can exclude the possibility that Thy-1 indirectly regulates tumor metastasis by the control of the infiltration of myeloid cells.

The tumor microenvironment, including fibroblasts and endothelial cells, plays a critical role in tumor progression. PECAM-1 on vascular EC promotes proliferation of melanoma cells and thus supports end-stage metastatic progression and tumor-induced cachexia. ${ }^{39}$ Moreover, angiogenesis is important for tumor growth as well as for the metastatic spread of malignant cells. ${ }^{19}$ Because neither primary tumor incidence and tumor growth nor angiogenesis was different in $T h y-1^{-/}$mice compared to controls, an indirect tumor-promoting effect by Thy-1 expression in the tumor microenvironment might not contribute to the control of metastases of melanoma cells by Thy- 1 .

As Thy-1 is highly expressed on T cells, we considered that alterations in T-cell immune response might be responsible for the reduced melanoma cell metastasis in Thy $-1^{-1-}$ mice. In accordance with Beissert et $\mathrm{al},{ }^{40}$ we did not see appreciable change in the number of $\mathrm{CD}^{+} \mathrm{T}$ cells or in the composition of leukocytes in the blood..$^{10}$ It has been reported that the differentiation of thymocytes, the number of antigen-presenting cells, the expression of co-stimulatory molecules on antigen-presenting cells, and the ability of dendritic cells to stimulate allogeneic $\mathrm{T}$ cells was only marginally affected in $T h y-1^{-/-}$mice. ${ }^{40,41}$ In a delayed type hypersensitivity model, a significantly reduced inflammatory infiltrate was seen, but there were no differences in the $\mathrm{CD} 4 / \mathrm{CD}^{+}$cells. ${ }^{40}$ Accordingly, we did not observe any detectable histological differences in $\mathrm{T}$-cell recruitment to tumor sites. T cells from Thy-1 ${ }^{-/}$ mice displayed similar proliferation after stimulation with concanavalin A, ionomycin, or PMA, whereas proliferation on CD3/CD28 stimulation was slightly reduced.$^{40}$ However, cytokine production by activated $\mathrm{T}$ cells was not affected in Thy $-1^{-/-}$mice. ${ }^{40}$ In the present study, activation and proliferation of $\mathrm{T}$ cells on anti-CD3/CD28 stimulation were not disturbed in Thy-1 $1^{-/-} \mathrm{T}$ cells. Taken together, lack of Thy-1 expression does not generally compromise the adaptive immune system. Therefore, altered T-cell functions do not appear to explain the decreased metastasis in Thy $-1^{-/-}$mice.

Previously, we reported that human Thy-1 mediates the adhesion of human melanoma cells to activated microvascular EC via the interaction with the $\alpha \mathrm{v} \beta 3$-integrin. ${ }^{12}$ Blocking of human Thy- 1 on EC clearly inhibited adhesion and transmigration of different $\alpha \mathrm{v} \beta 3$-positive melanoma cells to/through endothelial cells. ${ }^{12}$ The adhesive function of the murine Thy- 1 for the B16/F10 melanoma cells that were shown to express $\alpha v \beta 3$ integrin $^{42}$ was shown in adhesion assays. Consistent with our data, Jurisic et $\mathrm{al}^{26}$ demonstrated a Thy-1-dependent adhesion of B16/F10 melanoma cells to murine lymphatic EC. Considering the high expression of Thy- 1 on EC in human and experimental murine melanoma and its function as an adhesion molecule on EC for melanoma cells, the reduced metastases in Thy-1 $1^{-/-}$mice may be predominantly associated with a reduction of the Thy-1-mediated adhesion of melanoma cells to EC.

In conclusion, we showed that Thy- 1 is highly expressed on both activated lymphatic and vascular endothelial cells. VEGF and TNF- $\alpha$ were identified as potent inducers of Thy-1 expression. The physiological role of Thy-1 for lymphogenic and hematogenic metastasis of melanoma cells was underlined using experimental metastasis models in Thy $-1^{-/-}$mice. Thus, Thy- 1 contributes to metastasis of melanoma cells by mechanisms likely involving a decreased adhesion of melanoma cells to EC. 


\section{Acknowledgment}

We thank Heidi Gedicke for technical assistance.

\section{References}

1. Chaffer CL, Weinberg RA: A perspective on cancer cell metastasis. Science 2011, 331:1559-1564

2. Fidler IJ: The pathogenesis of cancer metastasis: the 'seed and soil' hypothesis revisited. Nat Rev Cancer 2003, 3:453-458

3. Rebhun RB, Cheng H, Gershenwald JE, Fan D, Fidler IJ, Langley RR: Constitutive expression of the alpha4 integrin correlates with tumorigenicity and lymph node metastasis of the B16 murine melanoma. Neoplasia 2010, 12:173-182

4. McGary EC, Lev DC, Bar-Eli M: Cellular adhesion pathways and metastatic potential of human melanoma. Cancer Biol Ther 2002, 1: 459-465

5. Voura EB, Ramjeesingh RA, Montgomery AM, Siu CH: Involvement of integrin alpha (v) beta(3) and cell adhesion molecule L1 in transendothelial migration of melanoma cells. Mol Biol Cell 2001, 12: 2699-2710

6. Bradley JE, Ramirez G, Hagood JS: Roles and regulation of Thy-1, a context-dependent modulator of cell phenotype. Biofactors 2009, 35:258-265

7. Haeryfar SMM, Hoskin DW: Thy-1: more than a mouse pan-T cell marker. J Immunol 2004, 173:3581-3588

8. Saalbach A, Wetzig T, Haustein UF, Anderegg U: Detection of human soluble Thy- 1 in serum by ELISA. Fibroblasts and activated endothelial cells are a possible source of soluble Thy-1 in serum. Cell Tissue Res 1999, 298:307-315

9. Saalbach A, Hildebrandt G, Haustein UF, Anderegg U: The Thy-1/Thy-1 ligand interaction is involved in binding of melanoma cells to activated Thy-1-positive microvascular endothelial cells. Microvasc Res 2002, 64:86-93

10. Schubert K, Polte T, Bonisch U, Schader S, Holtappels R, Hildebrandt G, Lehmann J, Simon JC, Anderegg U, Saalbach A: Thy-1 (CD90) regulates the extravasation of leukocytes during inflammation. Eur J Immunol 2011, 41:645-656

11. Wetzel A, Chavakis T, Preissner KT, Sticherling M, Haustein UF, Anderegg U, Saalbach A: Human Thy-1 (CD90) on activated endothelial cells is a counterreceptor for the leukocyte integrin Mac-1 (CD11b/CD18). J Immunol 2004, 172:3850-3859

12. Saalbach A, Wetzel A, Haustein UF, Sticherling M, Simon JC, Anderegg U: Interaction of human Thy-1 (CD 90) with the integrin alphavbeta3 (CD51/CD61): an important mechanism mediating melanoma cell adhesion to activated endothelium. Oncogene 2005, 24: $4710-4720$

13. Hermosilla T, Munoz D, Herrera-Molina R, Valdivia A, Munoz N, Nham SU, Schneider P, Burridge K, Quest AF, Leyton L: Direct Thy1/alphaVbeta3 integrin interaction mediates neuron to astrocyte communication. Biochim Biophys Acta 2008, 1783:1111-1120

14. Leyton L, Schneider P, Labra CV, Ruegg C, Hetz CA, Quest AF, Bron $\mathrm{C}$ : Thy- 1 binds to integrin beta(3) on astrocytes and triggers formation of focal contact sites. Curr Biol 2001, 11:1028-1038

15. Nosten-Bertrand M, Errington ML, Murphy KP, Tokugawa Y, Barboni E, Kozlova E, Michalovich D, Morris RG, Silver J, Stewart CL, Bliss TV, Morris RJ: Normal spatial learning despite regional inhibition of LTP in mice lacking Thy-1. Nature 1996, 379: 826-829

16. Vidal M, Morris R, Grosveld F, Spanopoulou E: Tissue-specific control elements of the Thy-1 gene. EMBO J 1990, 9:833-840

17. Huggenberger R, Ullmann S, Proulx ST, Pytowski B, Alitalo K, Detmar M: Stimulation of lymphangiogenesis via VEGFR-3 inhibits chronic skin inflammation. J Exp Med 2010, 207:2255-2269
18. Tsukamoto K, Hirata S, Osada A, Kitamura R, Shimada S: Detection of circulating melanoma cells by RT-PCR amplification of three different melanocyte-specific mRNAs in a mouse model. Pigment Cell Res 2000, 13:185-189

19. Emmett MS, Dewing D, Pritchard-Jones RO: Angiogenesis and melanoma - from basic science to clinical trials. Am J Cancer Res 2011, 1: 852-868

20. Zigrino P, Loffek S, Mauch C: Tumor-stroma interactions: their role in the control of tumor cell invasion. Biochimie 2005, 87:321-328

21. Bergenwald C, Westermark G, Sander B: Variable expression of tumor necrosis factor alpha in human malignant melanoma localized by in situ hybridization for mRNA. Cancer Immunol Immunother 1997, 44: 335-340

22. Hakansson A, Gustafsson B, Krysander L, Bergenwald C, Sander B, Hakansson L: Effect of interferon-alpha on the expression of tumour necrosis factor-alpha by metastatic malignant melanoma in vivo. Melanoma Res 1997, 7:139-145

23. Kiriakidis S, Andreakos E, Monaco C, Foxwell B, Feldmann M, Paleolog E: VEGF expression in human macrophages is NF-kappaBdependent: studies using adenoviruses expressing the endogenous NF-kappaB inhibitor IkappaBalpha and a kinase-defective form of the IkappaB kinase 2. J Cell Sci 2003, 116:665-674

24. Tugues S, Koch S, Gualandi L, Li X, Claesson-Welsh L: Vascular endothelial growth factors and receptors: anti-angiogenic therapy in the treatment of cancer. Mol Aspects Med 2011, 32:88-111

25. Forster R, Ohl L, Henning G: Lessons learned from lymphocytes: cC chemokine receptor-7 involved in lymphogenic metastasis of melanoma. J Natl Cancer Inst 2001, 93:1588-1589

26. Jurisic G, Iolyeva M, Proulx ST, Halin C, Detmar M: Thymus cell antigen 1 (Thy1. CD90) is expressed by lymphatic vessels and mediates cell adhesion to lymphatic endothelium. Exp Cell Res 2010, 316: 2982-2992

27. Ishizu A, Ishikura H, Nakamaru Y, Takeuchi E, Kimura C, Koike T, Yoshiki T: Thy-1 induced on rat endothelium regulates vascular permeability at sites of inflammation. Int Immunol 1995, 7:1939-1947

28. Ishizu A, Ishikura H, Nakamaru $\mathrm{Y}$, Kikuchi K, Koike T, Yoshiki T: Interleukin-1alpha regulates Thy-1 expression on rat vascular endothelial cells. Microvasc Res 1997, 53:73-78

29. Mason JC, Yarwood H, Tarnok A, Sugars K, Harrison AA, Robinson PJ, Haskard DO: Human Thy-1 is cytokine-inducible on vascular endothelial cells and is a signaling molecule regulated by protein kinase C. J Immunol 1996, 157:874-883

30. Takeuchi E, Ishikura $\mathrm{H}$, Ishizu A, Harada $\mathrm{H}$, Kato $\mathrm{H}$, Yasuda $\mathrm{K}$, Yoshiki T: Induced expression of Thy-1 molecules on dermal endothelial cells in skin allografts. Pathol Res Pract 1997, 193:623-627

31. Wetzel A, Wetzig T, Haustein UF, Sticherling M, Anderegg U, Simon JC, Saalbach A: Increased neutrophil adherence in psoriasis: role of the human endothelial cell receptor Thy-1 (CD90). J Invest Dermatol 2006, 126:441-452

32. Amatschek S, Kriehuber E, Bauer W, Reininger B, Meraner P, Wolpl A, Schweifer N, Haslinger C, Stingl G, Maurer D: Blood and lymphatic endothelial cell-specific differentiation programs are stringently controlled by the tissue environment. Blood 2007, 109: $4777-4785$

33. Pflicke H, Sixt M: Preformed portals facilitate dendritic cell entry into afferent lymphatic vessels. J Exp Med 2009, 206:2925-2935

34. Johnson LA, Clasper S, Holt AP, Lalor PF, Baban D, Jackson DG: An inflammation-induced mechanism for leukocyte transmigration across lymphatic vessel endothelium. J Exp Med 2006, 203:2763-2777

35. Johnson LA, Jackson DG: Inflammation-induced secretion of CCL21 in lymphatic endothelium is a key regulator of integrin-mediated dendritic cell transmigration. Int Immunol 2010, 22:839-849

36. Johnson LA, Jackson DG: Cell traffic and the lymphatic endothelium. Ann NY Acad Sci 2008, 1131:119-133

37. van Spriel AB, van Ojik HH, Bakker A, Jansen MJ, van de Winkel JG: Mac-1 (CD11b/CD18) is crucial for effective Fc receptor-mediated immunity to melanoma. Blood 2003, 101:253-258 
38. Yamada M, Yanaba K, Hasegawa M, Matsushita Y, Horikawa M, Komura K, Matsushita T, Kawasuji A, Fujita T, Takehara K, Steeber DA, Tedder TF, Sato S: Regulation of local and metastatic host-mediated anti-tumor mechanisms by L-selectin and intercellular adhesion molecule-1. Clin Exp Immunol 2006, 143:216-227

39. DeLisser H, Liu Y, Desprez PY, Thor A, Briasouli P, Handumrongkul C, Wilfong J, Yount G, Nosrati M, Fong S, Shtivelman E, Fehrenbach M, Cao G, Moore DH, Nayak S, Liggitt D, Kashani-Sabet M, Debs R: Vascular endothelial platelet endothelial cell adhesion molecule 1 (PECAM-1) regulates advanced metastatic progression. Proc Natl Acad Sci USA 2010, 107:18616-18621
40. Beissert S, He HT, Hueber AO, Lellouch AC, Metze D, Mehling A, Luger TA, Schwarz T, Grabbe S: Impaired cutaneous immune responses in Thy-1-deficient mice. J Immunol 1998, 161: 5296-5302

41. Hueber AO, Bernard AM, Battari CL, Marguet D, Massol P, Foa C, Brun N, Garcia S, Stewart C, Pierres M, He HT: Thymocytes in Thy-1-/mice show augmented TCR signaling and impaired differentiation. Curr Biol 1997, 7:705-708

42. Cowden Dahl KD, Robertson SE, Weaver VM, Simon MC: Hypoxiainducible factor regulates alphavbeta3 integrin cell surface expression. Mol Biol Cell 2005, 16:1901-1912 